\title{
Wakefield and Beam Centering Measurements of a Damped and Detuned X-Band Accelerator Structure*
}

\author{
C. Adolphsen, K. Bane, R. Jones, N. Kroll, D. McCormick, R. Miller, \\ M. Ross, T. Slaton and J.W. Wang \\ Stanford Linear Accelerator Center, Stanford University, Stanford CA 94309 \\ T. Higo \\ High Energy Accelerator Research Organization (KEK), Tsukuba, Japan
}

\begin{abstract}
In the Next Linear Collider (NLC) design, X-Band (11.4 GHz) accelerator structures are used to accelerate multibunch beams to several hundred GeV. Although these structures allow for high gradient operation, their strong deflecting modes impose a number of operational constraints. In particular, the long-range transverse wakefields generated by the bunches need to be reduced by about two orders of magnitude to prevent significant beam breakup. During the past five years, a reduction scheme that employs both detuning and damping of the structure dipole modes has been developed to meet this requirement. Several prototype Damped and Detuned Structures (DDS) have been built to test and refine this scheme. The wakefield of the latest version, DDS3, has recently been measured in the Accelerator Structure Setup (ASSET) facility at SLAC. In this paper, we present these results together with predictions based on an equivalent circuit model of the structure. We also present ASSET studies in which the beam-induced dipole signals that are coupled out for damping purposes are used to center the beam in the structure.
\end{abstract}

Presented at the 1999 Particle Accelerator Conference (PAC99), New York, NY, March 29 - April 2, 1999

\footnotetext{
* Work supported by Department of Energy contract DE-AC03-76SF00515.
} 


\title{
WAKEFIELD AND BEAM CENTERING MEASUREMENTS OF A DAMPED AND DETUNED X-BAND ACCELERATOR STRUCTURE *
}

\author{
C. Adolphsen" , K. Bane, R. Jones, N. Kroll, D. McCormick, R. Miller, M. Ross, T. Slaton, \\ J.W. Wang, SLAC, Stanford, CA, and T. Higo, KEK, Tsukuba, Japan
}

\begin{abstract}
We present wakefield measurements of a prototype Next Linear Collider (NLC) accelerator structure that was built with dipole mode damping and detuning to suppress the long-range transverse wakefield induced by a beam. In addition, we describe beam centering tests that use as a guide the dipole power coupled out of the structure for damping purposes.
\end{abstract}

\section{INTRODUCTION}

Nearly all designs being considered for future linear colliders employ multibunch operation to improve efficiency. A major concern in these designs is the potentially strong coupling of the bunch motions from the long-range transverse wakefields generated as the beams traverse the linac accelerator structures. For the NLC X-band (11.4 GHz) structures, two methods have been developed to reduce the wakefields to a manageable level. One method is mode detuning whereby the frequencies of the lowest band of dipole modes (near $15 \mathrm{GHz}$ ) are systematically varied along the structure to produce a Gaussian distribution in the mode density [1]. This detuning results in the destructive interference of the mode contributions, yielding an approximately Gaussian falloff in the net wakefield generated after each bunch. The structure design parameters are chosen to produce about a two orders of magnitude reduction by $1.4 \mathrm{~ns}$, the minimum bunch spacing being considered for the NLC.

This detuning works well to suppress the wakefield for about the first $30 \mathrm{~ns}$, after which its amplitude increases due to a partial recoherence of the mode excitations. This has led to the introduction of weak mode damping to offset this rise [2]. The damping is achieved through the addition of four single-moded waveguides (manifolds) that run parallel to the structure and couple to the cells through slots (see Fig. 1). When terminated into matched loads, the manifolds reduce the mode Q's from about 6000 to 1000 , enough to keep the wakefield from significantly increasing.

To date, three of these Damped and Detuned Structures (DDS) have been fabricated [3]. The cells for the most recent version, DDS3, were manufactured at LLNL [4] using single-diamond turning and then assembled in Japan using diffusion bonding [5]. This structure is $1.8 \mathrm{~m}$ long and contains 206 cells. Relative

\footnotetext{
${ }^{*}$ Work Supported by DOE Contract DE-AC03-76F00515.

\# Email: star@ slac.stanford.edu
}

to its predecessors, the rf match through the output ports of its manifolds is better. This improvement came in part from using vacuum waveguide couplers on the downstream ports instead of windows. Also, the dipole frequency profile was changed slightly to enhance the effect of the detuning. Instead of a Gaussian profile in the mode density, it was made in the product of the mode density and the mode coupling strengths to the beam. In addition, the sigma of the frequency distribution was decreased $(2.5 \%$ to $2.13 \%$ of $15.1 \mathrm{GHz})$, while the number of sigma was increased (4.0 to 4.78) to keep the frequency spread of the cells nearly the same.

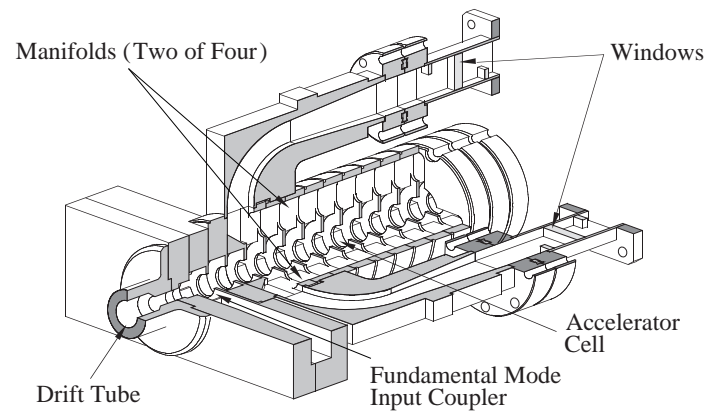

Fig. 1: Cutaway view of the upstream end of DDS3.

\section{WAKEFIELD MEASUREMENTS}

To measure the effect of the DDS3 changes on the wakefield, the structure was installed in the Accelerator Structure SETup (ASSET) facility, which is located in the upstream end of the main SLAC Linac. Here a positron beam induced wakefields in the structure that were 'witnessed' by an electron beam. Just downstream of the structure, a chicane of magnets steered the positrons (drive beam) to a dump and the electrons (witness beam) into the linac where their trajectory was measured. During the test, the eight manifold ports were connected via Heliax cables to processing electronics in the Klystron Gallery above the linac tunnel. The signals were either measured with a spectrum analyzer or downmixed to $310 \mathrm{MHz}$ and digitized to determine their amplitude and phase. A signal from a nearby stripline BPM served as the beam phase reference. Details of the wakefield and signal measurements can be found in references [6] and [7].

In brief, each measurement of the wakefield amplitude began by setting the relative beam timing to some multiple of the linac rf period $(350 \mathrm{ps})$. The relative timing was then stepped in $3 \mathrm{ps}$ increments to map the wakefield over a few oscillation periods $(\approx 200 \mathrm{ps})$. At 
each step, the drive beam position was moved parallel to the structure over a $+/-2 \mathrm{~mm}$ range and the resulting deflections of the witness beam recorded. The deflection angle per unit drive bunch offset was then computed and converted to a wakefield strength. Finally, the wakefield amplitude was obtained by fitting the wakefield strengths to a sine function with a frequency equal to the mean value for the lowest dipole band $(15.1 \mathrm{GHz})$.

The results from 35 such measurements over a $200 \mathrm{~ns}$ range are shown in Fig. 2. They are plotted versus the square-root of the relative beam time so values at short times are more discernible. Although the initial falloff of the wakefield is large, it is expected to stay below about $0.5 \mathrm{~V} / \mathrm{pC} / \mathrm{m} / \mathrm{mm}$ out to $20 \mathrm{~ns}$, instead of rising to values near $4 \mathrm{~V} / \mathrm{pC} / \mathrm{m} / \mathrm{mm}$ as is observed. This difference is likely the result of the cell fabrication errors being larger than the design tolerances. The effect of such errors are included in the predictions in Fig. 1, which were obtained from an equivalent circuit model of the structure [8]. In the top plot, the errors are based on limited cell QC measurements made prior to the structure assembly. They are essentially equivalent to random cell-to-cell, $5 \mathrm{MHz} \mathrm{rms}$, Gaussian frequency errors, and are larger than the $\approx 2 \mathrm{MHz}$ rms design tolerance. In the bottom plot, the errors were increased to best match the wakefield measurements, which required $12 \mathrm{MHz}$ rms values. Thus, the actual cell errors may be larger than that inferred from the QC results or more systematic in nature, which can enhance their
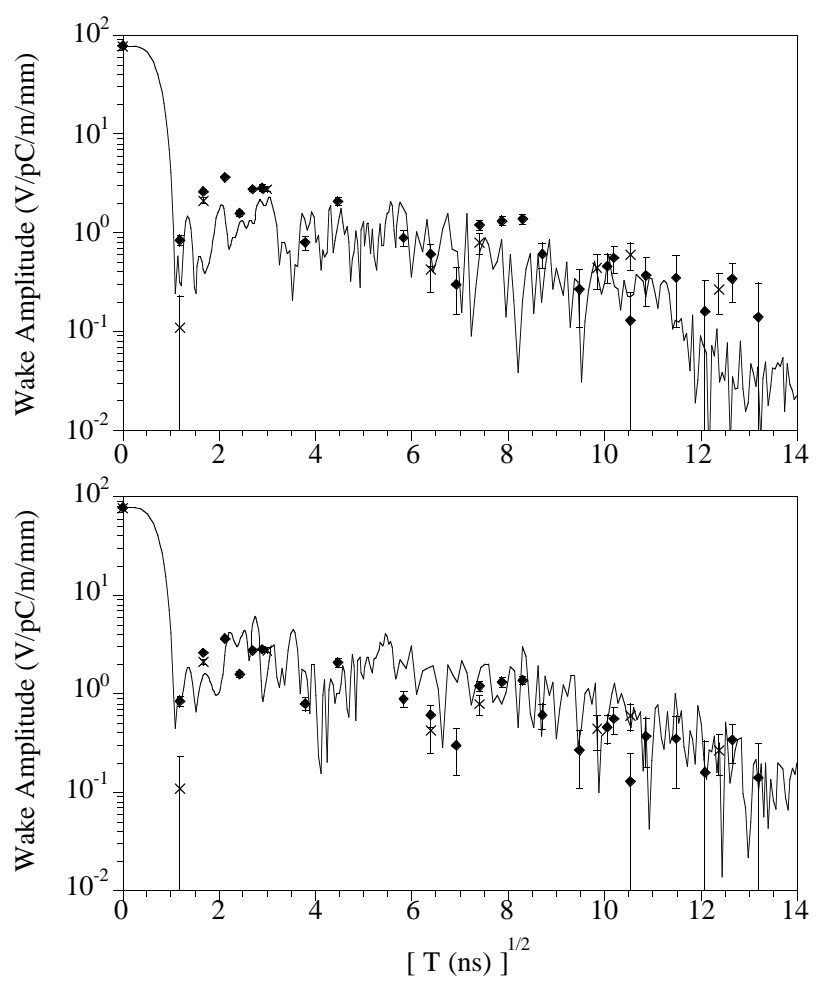

Fig. 2: Horizontal (crosses) and vertical (diamonds) wakefield measurements and predictions (solid lines) including $5 \mathrm{MHz}$ rms cell frequency errors (top) and $12 \mathrm{MHz}$ rms cell frequency errors (bottom). effect. The theory itself has worked well previously, yielding predictions in good agreement with the DDS1 measurements without added frequency errors [6].

The decrease in the wakefield that occurs at longer times is due to the mode damping. In contrast, the wakefield measured in a similar detuned-only structure slowly increases in time [9]. Another comparison of note is the similarity of the DDS3 horizontal and vertical results even though the upstream manifold ports for the horizontal modes were shorted for this test. This was done to assess the effect of removing the upstream ports in the future. However, the lack of a significant difference is not too surprising since the dipole mode power is expected to go mainly (99\%) to the downstream ports (this was experimentally verified as well). Finally, we note that the short-range $(<50 \mathrm{ps})$ DDS3 wakefield measurements agree well with a prediction that includes contributions from higher band modes [10].

\section{BEAM CENTERING}

Although the manifolds were nominally added to damp the dipole modes, they also yield signals that provide a measure the beam's transverse position in the structure. Moreover, the beam coupling to the modes is fairly localized ( 2 to 10 cells) so filtering the signals by frequency should yield beam offset information at different regions along the structure. The NLC goal is to use this signal information to keep the beams centered in the structures to about $+/-20 \mu \mathrm{m}$.

To evaluate these signals, data were taken in which the beam was stepped transversely across the structure while the signal from a $15 \mathrm{MHz}$ slice of the $14-16 \mathrm{GHz}$ dipole spectrum was processed. Figure 3 shows data taken at $15.0 \mathrm{GHz}$. As expected, a 180 degree phase transition occurs when the signal power goes through a minimum. The solid lines in the plots are fits to the data: a parabola to the power and an arctan function to the phase. In each case, a fit parameter was included to account for the addition of an out-of-phase signal component. The size of this component is conveniently expressed in terms of an equivalent beam offset, that is, the beam displacement relative to its position at minimum power that increases the dipole power by an amount equal to the out-of-phase power. The phase data yields the best measure of this offset, $5+/-2 \mu \mathrm{m}$. However, values as large as $100 \mu \mathrm{m}$ were initially observed. After some study, it was realized that an $\mathrm{X}-\mathrm{Z}$ correlation along the bunch $\left(\sigma_{\mathrm{Z}}=0.7 \mathrm{~mm}\right)$ was the likely source. This correlation was reduced by tuning the dispersive properties of the beamline to decrease the horizontal bunch width. Thereafter, the measured offsets were generally smaller than the NLC beam centering tolerance, which is desirable since it will simplify signal processing in the NLC.

Another parameter obtained from the fits to the dipole signal data is the beam position at the minimum 


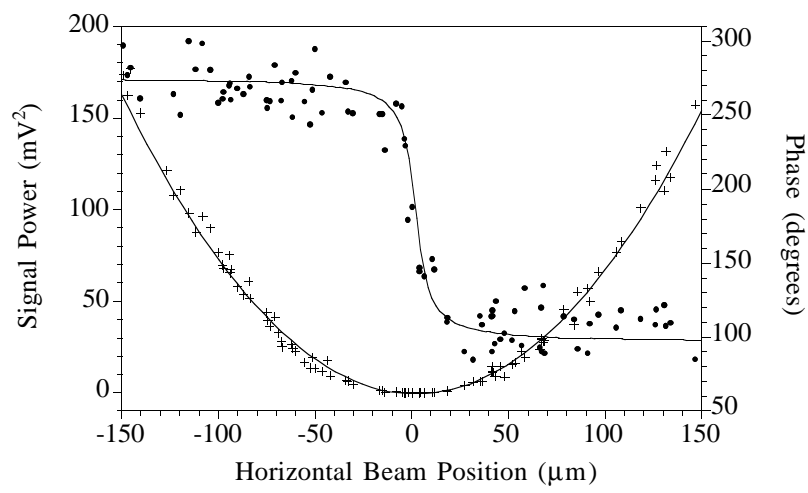

Fig. 3: $15 \mathrm{GHz}$ dipole signal power (pluses) and phase (circles) versus horizontal beam position. The fits to the data (solid lines) are described in the text.

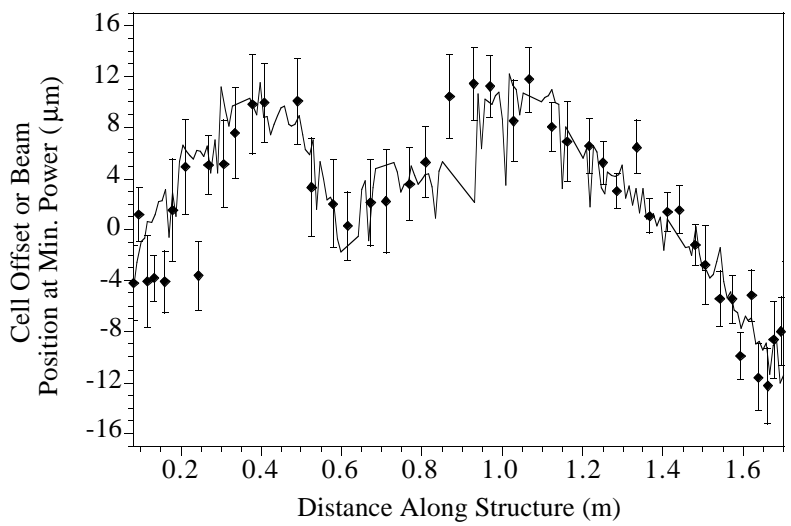

Fig. 4: Horizontal structure straightness measured with a beam (diamonds) and a CMM (solid line).

signal power. These values provide a measure of the structure straightness when plotted versus the corresponding points of origin of the signals (on average) along the structure. Figure 4 shows the horizontal straightness results that where derived using the frequency-to-cell mapping obtained from the equivalent circuit model of the structure [8]. Also shown are straightness results from a pre-installation survey of the outer surfaces of the cells $(30.5 \mathrm{~mm}$ radii) that was done with a Coordinate Measuring Machine (CMM). The good agreement between these measurements, and the micron-level local straightness of the structure, attest to the high precision with which the cells were fabricated and assembled. The vertical CMM and beam-based results agree less well, although a post-run survey of the structure indicates that it had vertically distorted by about $30 \mu \mathrm{m}$ since the initial survey.

From the straightness results, a beam orbit can be derived that should zero the integrated short-range transverse wakefield along the structure. To test how well this works, the ASSET drive beam was steered to an orbit based on the minimum dipole power results, and then the short-range wakefield $(T<200 \mathrm{ps})$ was measured with the witness bunch. In this case, an absolute wakefield was derived instead of one differential in the drive beam offset. For convenience, it was normalized to the differential short-range wake- field amplitude (i.e., the $\mathrm{T}=0$ point in Fig. 2). Hence, if the drive beam was offset by $20 \mu \mathrm{m}$, for example, the absolute wakefield should essentially be a sine wave with a $20 \mu \mathrm{m}$ amplitude. In practice, additional components of wakefield were observed that were independent of the drive beam position. Figure 5 shows two measurements in which these contributions are apparent. In the top plot, the $\mathrm{T}<50$ ps values are shifted negative for data that otherwise appear consistent with a beam offset of about $40 \mu \mathrm{m}$. The shape of the added wakefield is better seen in the bottom plot where the beam was likely centered to $<10 \mu \mathrm{m}$. At this level, a sine-like $21 \mathrm{GHz}$ component also becomes apparent. The $\mathrm{T}<50 \mathrm{ps}$ component is thought to originate from wakefields generated upstream of ASSET since it was also observed in measurements of a C-Band structure [11]. The origin of the $21 \mathrm{GHz}$ component is not known, but it would have little impact on NLC operation. Despite these complications, the attempts to center the drive beam generally yielded offsets $<20 \mu \mathrm{m}$ based on the size of the $15.1 \mathrm{GHz}$ component. Thus, these techniques appear promising for meeting the NLC beam centering requirements.

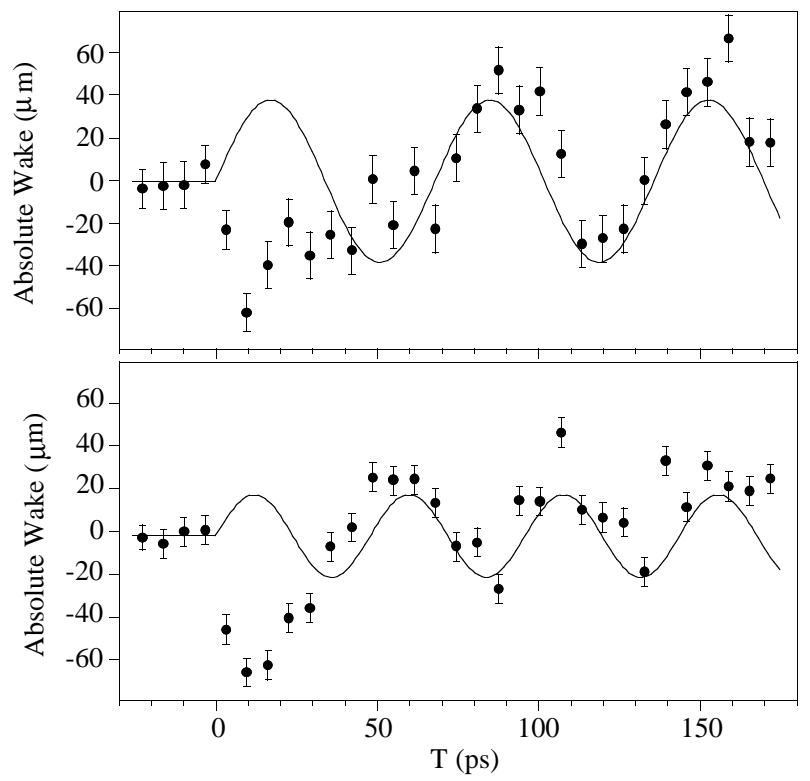

Fig. 5: Vertical absolute wakefield measurements made as part of the beam centering tests. The solid lines are $15.1 \mathrm{GHz}$ (top) and $21 \mathrm{GHz}$ (bottom) sine wave fits to the $\mathrm{T}>50 \mathrm{ps}$ data.

\section{REFERENCES}

[1] K. A. Thompson et al., SLAC-PUB-6032 (Nov. 1993).

[2] N. M. Kroll, SLAC-PUB-7541 (May 1997).

[3] J.W. Wang et al., FRA18, these proceedings.

[4] J. Klingmann et al., MOP51, these proceedings.

[5] T. Higo et al., FRA16, these proceedings.

[6] C. Adolphsen et al., SLAC-PUB-7519 (May 1997).

[7] M. Seidel, SLAC-PUB-7557 (June 1997).

[8] R. Jones, private communication.

[9] C. Adolphsen et al., SLAC-PUB-6629 (Aug. 1994).

[10] K. Bane, private communication.

[11] T. Shintake, FRA14, these proceedings. 